\title{
Development of the Endocardium
}

\author{
Ian S. Harris • Brian L. Black
}

Received: 7 January 2010/Accepted: 17 January 2010/Published online: 5 February 2010

(C) The Author(s) 2010. This article is published with open access at Springerlink.com

\begin{abstract}
The endocardium, the endothelial lining of the heart, plays complex and critical roles in heart development, particularly in the formation of the cardiac valves and septa, the division of the truncus arteriosus into the aortic and pulmonary trunks, the development of Purkinje fibers that form the cardiac conduction system, and the formation of trabecular myocardium. Current data suggest that the endocardium is a regionally specialized endothelium that arises through a process of de novo vasculogenesis from a distinct population of mesodermal cardiogenic precursors in the cardiac crescent. In this article, we review recent developments in the understanding of the embryonic origins of the endocardium. Specifically, we summarize vasculogenesis and specification of endothelial cells from mesodermal precursors, and we review the transcriptional pathways involved in these processes. We discuss the lineage relationships between the endocardium and other endothelial populations and between the endocardium and the myocardium. Finally, we explore unresolved questions about the lineage relationships between the endocardium and the myocardium. One of the central questions involves the timing with which mesodermal cells, which arise in the primitive streak and migrate to the cardiac crescent, become committed to an endocardial fate. Two competing conceptual models of endocardial specification have been proposed. In the first, mesodermal precursor cells in the cardiac crescent are prespecified to become either endocardial or myocardial cells, while in the second, fate plasticity is retained by bipotential cardiogenic cells in the
\end{abstract}

I. S. Harris · B. L. Black $(\square)$

Cardiovascular Research Institute, University of California,

San Francisco, 600 16th Street, Mail Code 2240,

San Francisco, CA 94158-2517, USA

e-mail: brian.black@ucsf.edu cardiac crescent. We propose a third model that reconciles these two views and suggest future experiments that might resolve this question.

Keywords Endocardium - Endothelial $\cdot$ Heart

\section{Overview of Cardiogenesis}

The cardiovascular system is the first organ system to form and function in the vertebrate embryo. The heart forms when regions of bilaterally symmetrical cardiac progenitor cells in the anterior lateral mesoderm fuse at the ventral midline to form a linear tube, which is continuous with the dorsal aorta anteriorly and the cardinal veins posteriorly [5]. The heart tube at the time of its formation is a twolayered structure, composed of an inner endothelial layer and an outer myocardial layer. The myocardial layer will form the muscular walls of the heart and the muscular portions of the interventricular septum. The endothelial layer comprises the endocardium [1, 4, 6, 54].

In addition to forming an endothelial layer within the heart that is contiguous with the rest of the vasculature, the endocardium has several other essential functions during heart development. For instance, the endocardium plays an important role in signaling to cardiomyocytes and is required for proper formation of trabecular myocardium $[60,65]$. It is also involved in the signaling required for transdifferentiation of myocytes into the Purkinje fiber cells that comprise much of the cardiac conduction system [41, 42]. In addition, cells from specific regions of the endocardium will undergo endocardial-to-mesenchymal transformation (EMT) to form the endocardial cushions [12]. The endocardial cushions give rise to several important structures within the heart, including the valve 
leaflets, the membranous portion of the interventricular septum, and the atrial septum [51, 57]. In addition, endothelial-derived cells in the cardiac cushions function, along with cells of neural crest origin, to separate the common outflow tract (OFT) into the pulmonary artery and aorta $[27,38,57]$. Despite the many essential functions of the endocardium during development and in postnatal cardiac function, much remains to be learned about the development and embryonic origins of the endothelial progenitor cells that will give rise to the endocardium.

\section{Specification of Endothelial Cells}

Vasculogenesis, the de novo formation of blood vessels, occurs necessarily before the onset of blood circulation. In amniotes, endothelial and hematopoietic cells first appear at the same time in the extraembryonic yolk sac and are thought to arise from a common mesodermal precursor, the hemangioblast [48, 67]. Vasculogenesis begins when hemangioblasts coalesce to form blood islands. The outer cells in the blood islands flatten as they differentiate into endothelial cells, while the inner cells differentiate into hematopoietic progenitors $[20,50]$. Ultimately, these cells will form the extraembryonic yolk sac vasculature and the blood cells contained within it [20].

De novo vasculogenesis also occurs at multiple discrete sites within the embryo proper, including the paired dorsal aortae, within the heart tube, in the neural plexus, and in the primary vascular plexus $[14,20,50]$. In the embryo, mesoderm-derived endothelial precursors called angioblasts aggregate to form the dorsal and ventral aortae and the vitelline vessels [20]. Endocardial precursors arise via a similar process of de novo vasculogenesis within the cardiac crescent and are subsequently arranged into a vascular tube during ventral morphogenesis $[1,28]$. The precise timing of specification of these precursors remains incompletely understood and is discussed below.

Multiple studies in fish, birds, and mammals have suggested an intimate relationship between endothelial and hematopoietic lineages. Lineage-tracing studies performed in zebrafish have revealed that blood cells and endothelial cells are derived from common precursors termed hemangioblasts in the ventral marginal zone of the blastula [33, 58]. In the mouse, hemangioblasts have been identified by coexpression of the mesodermal gene brachyury (Bry) and the VEGF receptor, Flk-1 [18]. The relationship between vasculogenic and hematopoietic precursors has recently been found to be even more complex: genetic fate-mapping studies in the mouse revealed that an endothelial-specific Cre transgene resulted in the genetic labeling of a large number of hematopoietic cells in the adult bone marrow [69]. These findings lend support to the concept, previously suggested by work in avian embryos, of a population of hemogenic endothelial cells that have the capacity to give rise to blood $[29,69]$.

After formation of the embryonic and extraembryonic vasculature, the initial primitive vessels are extensively remodeled into mature vessels through a complex process termed angiogenesis [20, 49, 50]. In addition, smooth muscle cells or pericytes are recruited to the vessel walls from surrounding mesenchyme [24]. During angiogenesis and vascular remodeling, vessels expand, coalesce, branch, and become specialized as arteries, veins, and capillaries $[20,24,25]$.

\section{Embryonic Origin of the Endocardium and Its Relationship to Other Endothelial Cells}

The unique location and specialized functions of the endocardium have led some investigators to question whether it represents a unique and molecularly distinct population of cells or rather simply a spatially restricted subpopulation of endothelium. Several genetic models have offered some insight into this question. Tie and Tie2, two structurally related receptor tyrosine kinases, are required throughout the vasculature during late organogenesis and during postnatal life in mice [63]. Tie ${ }^{-/-} ;$Tie $^{-/-}$embryos display normal extracardiac vasculogenesis but have profound cardiac defects, including deficient endocardium, and $\mathrm{Tie}^{-/-} ; \mathrm{Tie} 2^{-/-}$endothelial cells contribute normally to all vascular structures except the endocardium [52]. Similar results were observed in cloche mutant zebrafish, which have structurally normal endothelium of the dorsal aortae and cardinal veins, despite the absence of the endocardium [59]. Notably, cloche is also required for formation of hematopoietic stem cells, as well as for formation of the endocardium [59]. Importantly, and in contrast to the above examples, no factors or genes have been identified to date to be dispensable for endocardial specification but to be required for endothelial specification elsewhere in the embryo, suggesting that endocardial progenitors comprise a spatially restricted subset of a larger endothelial precursor population.

The endocardium appears to be unique among endothelial tissues in that it arises through de novo vasculogenesis from a population of precardiac progenitors, marked by Flk1 expression in the late primitive streak, which also give rise to cardiomyocytes [30]. Recent work has refined our understanding of the clonal origins of the endocardium. Embryonic stem cells derived from an Nfatcl-nuc-lacZ bacterial artificial chromosome (BAC) transgenic mouse line were used to generate embryoid bodies, which could be separated into three distinct populations on the basis of expression levels of E-cadherin (ECD) and Flk1: 
$\mathrm{ECD}^{\text {high }} \mathrm{Flk} 1^{\text {low }}, \mathrm{ECD}^{\text {low }} \mathrm{Flk} 1^{\text {high }}$, and $\mathrm{ECD}^{\text {low }} \mathrm{Flk} 1^{\text {low }}$ [43]. The ECD ${ }^{\text {low }} \mathrm{Flk} 1^{\text {high }}$ population represents the hemangioblast population [43]. Importantly, only the $\mathrm{ECD}^{\text {low }} \mathrm{Flk} 1^{\text {low }}$ population demonstrated detectable X-gal staining (indicative of $\mathrm{Nfatcl}$ expression), suggesting that the endocardium is derived from this non-hemangioblast population [43]. Interestingly, the $\mathrm{ECD}^{\text {low }} \mathrm{Flk} 1^{\text {low }}$ population also displayed markers of vascular differentiation, including $\mathrm{Cdh} 5$ (VE-cadherin) and CD31 (Pecam1) and markers of myocardial differentiation, including $N k x 2-5, \beta-M y H C, c T n T$, GATA4, and Mef2c [43], again supporting the notion that this population gives rise to both myocardial and endocardial cells. These data are consistent with the recent discovery that the hemangioblast and cardiac potential of embryonic stem (ES) cell-derived embryoid bodies segregated to two distinct populations, $\mathrm{Bry}^{+} / \mathrm{Flk}^{+}$and $\mathrm{Bry}^{+} /$ Flk $1^{-}$, respectively, based on cell surface marker expression [32]. It was later found that the $\mathrm{Bry}^{+} / \mathrm{Flk}^{-}$population generates a second $\mathrm{Flk} 1^{+}$population with robust cardiac potential that contains progenitors of cardiomyocytes, vascular smooth muscle cells, and endothelial (endocardial) cells [17]. Taken together, these data suggest that endocardial cells arise, at least in part, from a cardiovascular progenitor population distinct from the hemangioblasts that give rise to other endothelial subtypes.

The endocardium is known to express lineage-specific markers in a pattern distinct from other endothelial cell types. For example, $\mathrm{Nfatcl}$ is specifically expressed in the endocardium, with transcripts detectable at the time of initiation of endocardial differentiation in the primary heart-forming field at E7.5 and intracellular protein detectable at the linear heart tube stage at E8.5 [13]. Interestingly, despite its intranuclear location during early endocardiogenesis, NFATc1 does not appear to be required for endocardial specification or proliferation, although its activity is critical during later cardiac development and valvulogenesis $[13,53]$. Thus, all of the observations to date support a model in which the endocardium is a spatially restricted population of endothelium, arising as a result of de novo vasculogenesis from precursor cells present in the cardiac crescent. Furthermore, the observations to date suggest that highly related, but distinct, genetic programs, involving overlapping subsets of factors, control the development of the endothelial cells that will comprise the endocardium versus endothelial cells located elsewhere in the embryo.

\section{Relationship Between Endocardium and Myocardium}

The lineage relationship between endocardial and myocardial cells remains unresolved. Specifically, while it is clear that endocardial and myocardial cells share a common progenitor, it is not clear whether these progenitors are present in the early heart field within the anterior lateral mesoderm. Two basic models of lineage determination in the heart have been proposed [16, 28, 35]. The first model is based primarily on observations in avian and fish embryos and postulates that cells in the cardiac mesoderm are prespecified in the primitive streak, prior to migration to the cardiac crescent, to become either myocardium or endocardium (Fig. 1) [11, 66]. The second model is largely based on work performed in mouse embryos or using cells derived from mammalian embryos and suggests that endocardial and myocardial cells share a common progenitor within the cardiac mesoderm and that these bipotential (or multipotential) cells maintain fate plasticity until a much later time in development than the prespecification model (Fig. 2) [7, 30, 39, 46].

\section{Gastrulation}

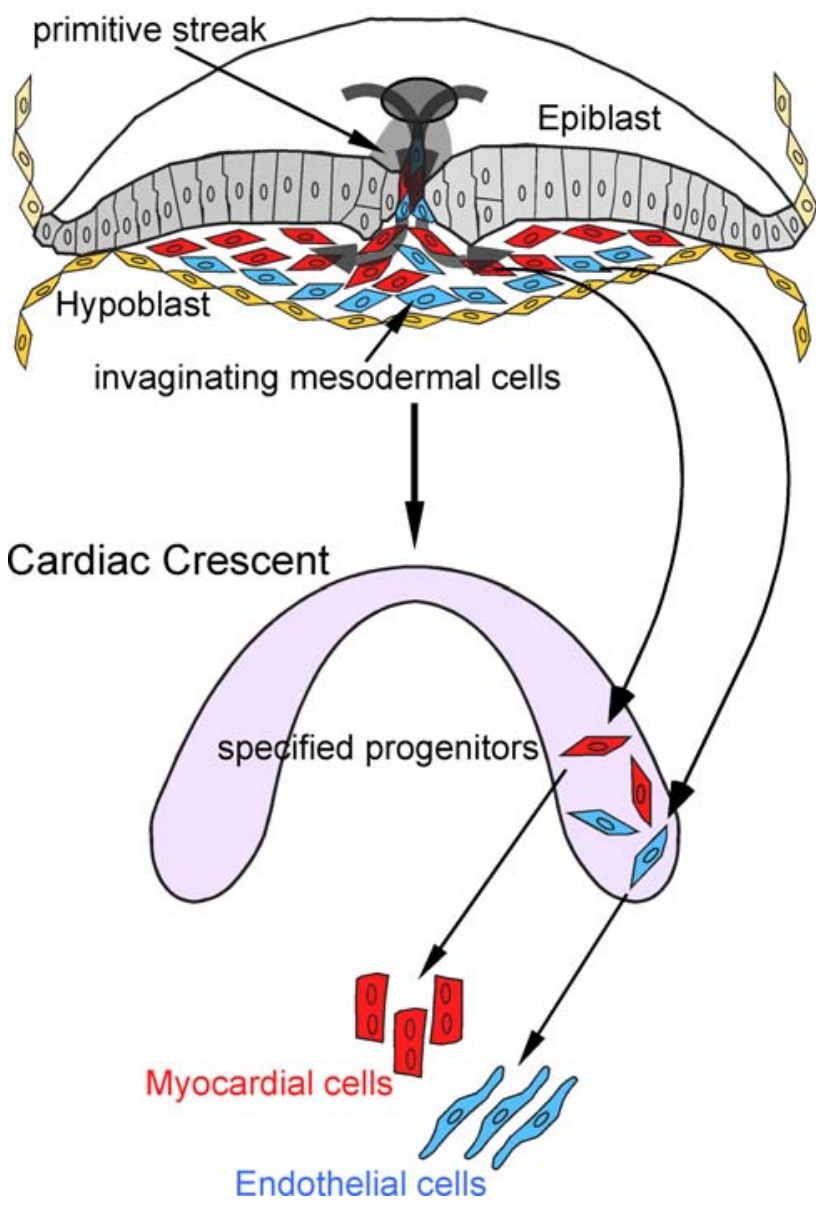

Fig. 1 A prespecification model for endocardial cells. Data obtained primarily from avian and fish models suggest that mesodermal cells are prespecified in the primitive streak before migration to the cardiac crescent to adopt either an endocardial or a myocardial fate. Endocardial precursors then receive inductive cues from the underlying endoderm to differentiate into cells with an endothelial phenotype 


\section{Gastrulation}

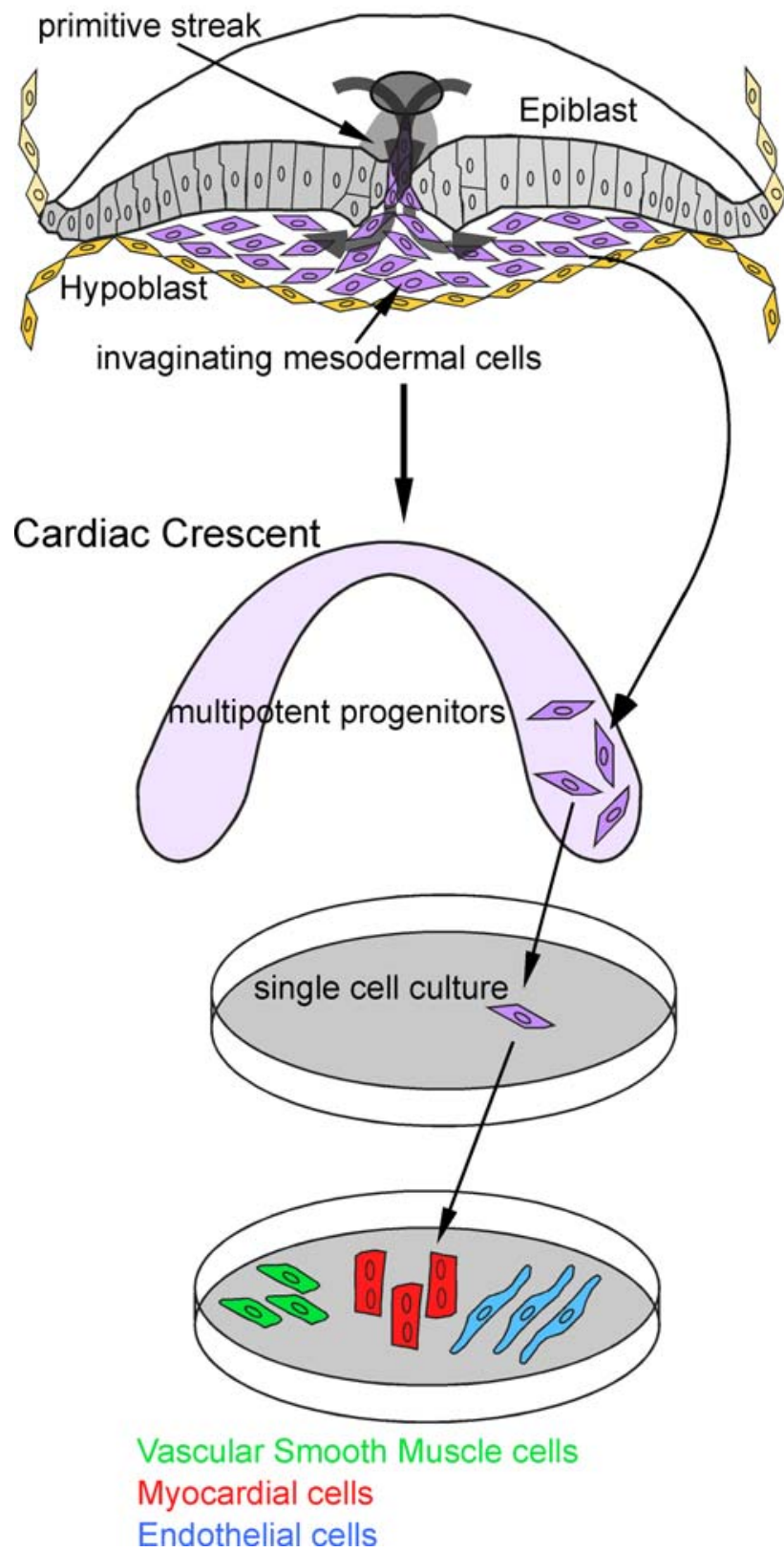

Fig. 2 A model for multipotent progenitor cells in the cardiac crescent. Data acquired primarily from mouse fate-mapping studies and ex vivo approaches using cells from mouse embryos suggest that there are multipotential progenitors in the cardiac crescent that can then be specified to adopt endocardial, myocardial, or vascular smooth muscle cell fates

\section{Model 1: Cardiogenic Precursors Are Prespecified When They Migrate from the Primitive Streak to the Cardiac Crescent}

In an elegant set of lineage-tracing experiments, Mikawa and coworkers introduced $\beta$-gal-expressing viral particles into the mesodermal layer of gastrulating chick embryos at
Hamburger-Hamilton $(\mathrm{HH})$ stage 4 (11). When the embryos were examined at $\mathrm{HH}$ stage $10, \beta$-gal ${ }^{+}$cells were seen in both the endocardium and the myocardium. However, individual patches of $\beta$-gal ${ }^{+}$cells, thought to have arisen from individual infected precursors, were seen to contain either myocardial or endocardial cells, but never both [11]. The latter result was observed even at viral titers that would only result in the infection of individual cells. From these results, Mikawa and coworkers concluded that individual cells in the cardiac mesoderm were already specified to give rise either to myocardial cells or to endocardial cells [11]. In a follow-up set of similar experiments, the same group infected cells in the primitive streak at $\mathrm{HH}$ stage 3 with a $\beta$-gal-encoding replicationdeficient virus. Again, when they examined embryos at $\mathrm{HH}$ stage 10 , they observed similar results as with the previous set of experiments. Individual $\beta$-gal ${ }^{+}$patches of labeled cells, presumably derived from just a single infected progenitor cell, contained either endocardial cells or myocardial cells, but never both [66]. As with their previous study, these results support a model in which precardiac mesoderm cells are prespecified to either the myocardial or the endocardial lineage prior to migration through the primitive streak (Fig. 1).

Similarly, in a series of cell-labeling experiments in zebrafish, it was found that the majority of cells within the cardiogenic region did not give rise to endocardial cells. Rather, a subset of mesoderm precursors located in the ventral region of the heart field was found to give rise to endocardial cells [33]. As was observed in the chick experiments described above, individual cells labeled in the heart field never gave rise to both myocardium and endocardium.

\section{Model 2: Precardiac Mesoderm in the Cardiac Crescent Contains Multipotential Cells That Can Give Rise to Both Endocardium and Myocardium}

In contrast to the studies described above, in which endocardial cells are prespecified in the cardiac mesoderm, studies performed primarily in mouse embryos have suggested a different model in which mesodermal precursor cells in the cardiac crescent maintain fate plasticity and can give rise to both myocardium and endocardium [39]. Molecular marker analyses and Cre-based fate mapping approaches in mice have resulted in the identification of markers of the cardiac lineage that begin to be expressed in the mesoderm of the cardiac crescent after the cells have migrated through the primitive streak. Several of these markers label cells that give rise to descendents present in both the myocardium and the endocardium [9, 39, 64]. 
A lineage analysis of Isll-expressing cells in developing mouse embryos demonstrated that $I s l I^{C r e}$-marked cells, which are first apparent in the cardiac crescent, give rise to both myocardial and endocardial cells [9]. This observation supports the notion that these two cell types arise from a common progenitor in the cardiac crescent, although it does not exclude the alternative hypothesis that Isll may be simultaneously expressed in distinct progenitors for each of these lineages. Similarly, Verzi et al. examined the activity of a Cre-dependent reporter in which Cre was expressed under the control of anterior heart field (AHF)-specific regulatory elements from the $M e f 2 c$ gene and found that both endocardial and myocardial cells were marked by the activity of the transgene [64]. Again, this is consistent with the notion that these two tissues share a common progenitor in the cardiac crescent but allows the possibility that the activity of the Mef2c-AHF-Cre transgene might also independently mark two discrete progenitor populations. Likewise, Chien and colleagues used genetic fate-mapping techniques in mice to identify a subset of multipotent cardiovascular progenitors with the transcriptional signature $I s l 1^{+} ; \mathrm{FlkI}^{+} ; \mathrm{Nkx2-5^{+ }}$ that gave rise to multiple cell types in vivo and were able to generate cardiac muscle, smooth muscle, and endothelial cells in vitro (Fig. 2) [44].

More recent work has further elucidated the lineage relationships between myocardium and endocardium in the mouse. Nkx2-5 is one of the earliest markers of the myocardial lineage, first expressed at the cardiac crescent stage [22, 23, 31, 36, 56]. Germline deletion of $N k x 2-5$ results in profound cardiac defects, characterized in part by complete absence of the endocardial cushions [37, 62]. These observations, combined with the fate-mapping and lineage analyses discussed in the previous paragraph, provide further evidence for a model in which myocardial and endocardial cells arise from a common progenitor cell in the precardiac mesoderm [35]. Alternatively, these data are also consistent with the notion that $N k x 2-5^{+}$progenitor cells in the cardiac crescent do not represent a uniform population of bipotential cells but, rather, are composed of at least two distinct subpopulations that have been prespecified to adopt myocyte and endocardial fates but that cannot be resolved on the basis of known markers. This alternative interpretation of the majority of these mouse experiments would be more consistent with the chick and zebrafish data supporting prespecification of endocardial and myocardial progenitors within the precardiac mesoderm, as discussed in the last section.

Further support for the notion that cardiac progenitor cells in the crescent remain multipotential and give rise to both myocytes and endocardial cells is provided by recent work examining the regulation of the Ets-related protein Etv2 (Etsrp71, ER71) gene [19]. Etv2 has been identified recently as a major master control gene for endothelial specification $[15,19,34,61]$. Interestingly, Etv2 regulation in the endocardium appears to be under direct control of Nkx2-5, a cardiac master regulator $[2,19,36]$. Although these results do not directly address the potential or fate of progenitors within cardiogenic mesoderm, the observation that a cardiac master regulator activates an endothelial master regulator is consistent with the idea that an early program involving Nkx2-5 sets up both the endocardial and the myocardial programs and suggests that the endocardium arises secondarily from a subset of cardiogenic progenitors via a cell-autonomous effect of Nkx2-5.

Because of the limited number and relative inaccessibility of cardiogenic mesoderm cells in the early mouse embryo, ES cell-based models have also been used extensively to investigate the early fate decisions of cardiogenic mesoderm. Kattman and colleagues examined in vitro differentiation of an ES cell line with a GFP reporter gene targeted to the mesoderm specification gene brachyury $[18,26,30]$. As ES cells were differentiated, some expressed GFP, indicating that they had adopted a mesoderm fate. These GFP-positive cells were all initially $\mathrm{Flkl}^{-}$, but a subset induced expression of the endothelial marker gene $F l k l$ and this induction could be augmented by addition of the endothelial growth factor VEGF to the culture medium [30]. Importantly, some $F l k 1^{+}$cells were observed to form aggregates of beating cells that expressed the cardiac-specific marker cTnT, indicating that these $F l k 1^{+}$cells had the capacity to form either endothelial cells or cardiomyocytes [30].

In another study, Masino and colleagues used fluorescence-activated cell sorting (FACS) to isolate cells expressing YFP under control of an enhancer element from the $N k x 2-5$ locus at the cardiac crescent stage, at the linear heart tube stage, and during cardiac looping, and they performed transcriptome-wide expression profiling [39]. These studies showed that these $N k x 2-5^{+}$cardiac precursor cells expressed markers of both cardiac myocyte differentiation and endothelial differentiation, lending credence to the notion that the precardiac mesoderm contains multipotential cells with the capacity to give rise to at least two descendent types, myocardial and endothelial cells [39].

It is important to note that cells with the capacity to give rise to both endothelial and myocardial cells are not restricted to mammalian systems. Several laboratories have also found evidence of bipotential mesoderm precursors in avian cells as well. The QCE-6 cell line, derived from explants of Japanese quail embryos collected at $\mathrm{HH}$ stage 4 , can differentiate into both endothelial and myocardial cells [55], suggesting that a degree of plasticity exists in those progenitor cells as well. In addition, overexpression of the bHLH transcription factor Scl/Tal1 together with the Lim-homeodomain transcription factor Lmo2 in early zebrafish embryos has been shown to result in an expansion 
of endothelial and hematopoitic cells, at the expense of myocardial differentiation, suggesting a shared precursor population [21].

Thus, it remains unclear whether mesodermal cells in the cardiac crescent represent a homogeneous population of bipotential cardiac progenitors or, rather, a heterogeneous population of progenitors that have been prespecified to adopt either a myocardial or an endocardial fate. Future studies will be required to determine whether these two models can be reconciled and to clarify this issue.

\section{Transcription Factors Involved in Endocardial Development}

Regardless of whether endocardial progenitors are prespecified at the time they enter the cardiac crescent or whether cells maintain significant plasticity until later in development, it is clear that the endocardium develops from a subset of cardiogenic precursors within the anterior lateral mesoderm [43]. In order to understand how these cardiogenic cells enter either the myocardial or the endocardial fate, it is critical to examine the transcriptional networks involved in these fate decisions. While endocardial cells arise from a distinct population of cardiovascular precursors, their specification appears to involve a genetic program shared with other endothelial populations. Several transcription factors have been shown to be required for both endothelial and endocardial specification. For example, Etv2 and Scl/Tal1, both key early regulators of endothelial development, are also required for endocardial specification [8, 14, 19]. These observations support the view that the endocardium is simply a spatially restricted subpopulation of endothelium. However, a limited number of transcription factors are expressed specifically in the endocardium, and examination of these and their relationship to established cardiac programs has led to an enhanced understanding of the unique nature of the endocardium.

The GATA family of zinc-finger proteins has been shown to be critical for cardiac development [10]. Al Moustafa and Chalifour found that mesodermal cells could be differentiated in vitro by treatment with retinoic acid [3]. Upon treatment, these cells attenuate expression of the pan-mesoderm markers Brachyury and GATA4 and begin to express GATA5 [47]. At the same time, the transcription factor NFATc1 is translocated to the nucleus, where it can participate in transcriptional activation[47]. In vitro transactivation assays using a GATA-responsive reporter construct showed that GATA5 and NFATc1 synergistically activate endocardial gene expression [47]. This cooperative activation by GATA and NFAT factors lends support to the notion that the endocardium develops within a field of cardiac progenitors as a molecularly distinct endothelial subpopulation in response to cardiac-specific cues.

Etv2 is an important endothelial and hematopoietic determination factor and has been shown recently to play a fundamental role in the transcriptional program controlling development of the endothelium, including the endocardium [15, 19, 34, 61]. Etv2-null embryos are normal at E8.5 but are nonviable between E9.0 and E10.0 and have no identifiable endocardium or endothelium [19]. Interestingly, Etv2 appears to be activated directly by Nkx2-5 in the progenitors that will give rise to the endocardium [19]. This result suggests that myocardial specification (or a more general specification of cardiac progenitors) may precede endocardial specification and Etv2 activation. Etv2, in turn, bound to the promoter region of the Tie 2 gene in vivo and transactivated it in culture [19]. These data suggest that, while sharing a basic transcriptional network with other endothelial types, the endocardium is unique in that it arises from a distinct cardiac precursor population and that the genetic program controlling its specification is subordinate to a cardiogenic program in a transcriptional hierarchy responsible for cardiovascular development.

\section{Future Directions}

As outlined above, a central question in understanding endocardial development is whether the fate of precursor cells in the mesoderm is prespecified by the time they arrive in the cardiac crescent or whether these precursors retain some fate plasticity. On the whole, in vivo avian models have supported the prespecification model, while in vitro systems, primarily using mammalian cells, have lent support to the plasticity model [66]. A shortcoming of the existing in vivo work in mammalian systems is that it has failed to exclude the possibility that available markers might not be able to resolve potential component subpopulations within the cardiac crescent. A trivial explanation for discrepancies between the two models is that there may be inherent differences in the model organisms. However, another way of reconciling the two models is to postulate that cardiogenic precursors do indeed retain plasticity within the cardiac crescent, but that their fate decisions once they arrive in the crescent are constrained by positional cues. In this way, bipotential precursor cells labeled in situ will be seen giving rise to only one cell type, but can be induced to differentiate into both endocardium and myocardium when removed from the embryo (and the corresponding positional cues within the embryo) and cultured ex vivo.

To address the issue of whether bipotential (or multipotential) progenitor cells within the nascent heart field are restricted in their fate due to positional information, 
cells from the ventral region of the heart field could be labeled with 1,1'-dioctadecyl-3,3,3',3'-tetramethylindocarbocyanine perchlorate (diI) and transplanted to a more dorsal position in chick embryos. Subsequent identification of the label in myocardial cells would suggest that the cardiac crescent contains bipotential progenitors whose fate determination is constrained by positional cues. Additionally, the role of positional cues and possible species differences could be addressed in mouse embryos by retrospective fate mapping, as has been done so elegantly to map contributions of myocardial progenitors to the heart [40]. Specifically, an nlaacZ reporter construct could be targeted to the $N k x 2-5$ locus in mouse. As has been shown previously, this reporter contains a lac $Z$ reporter gene with a frameshift mutation (hence the name laacZ) and is inactive unless a rare intragenic recombination event occurs [40]. In the event of such a recombination, which restores functional lac $Z$ expression, all progeny of the cell in which recombination occurred will be labeled [40]. Clonal labeling of both endocardial and myocardial cells in the neonatal heart would provide compelling evidence that individual $N k \times 2$ $5^{+}$precursors in the cardiac crescent can give rise to both lineages.

Additionally, a genetic approach could be taken to address the possibility that a subset of cardiovascular progenitors becomes specified in the cardiac crescent to adopt an endothelial cell fate under the control of the cardiac regulator $\mathrm{Nkx2-5}$. A transgenic mouse line has been published that expressed Cre recombinase under the control of an enhancer element from the $N k x 2-5$ locus $[45,68]$. Cre expression in these mice is first detected in cardiovascular progenitors of the cardiac crescent and is restricted to the myocardium after cardiac looping [68]. This Cre transgenic line could be used to create a conditional deletion of Etv2. If such a deletion resulted in disruption of the endothelial program in these cardiovascular progenitors and a defect in the endocardium, it would clarify two of the central questions of endocardial development. First, it would lend support to the notion that the cardiac master regulator Nkx2-5 and the endothelial master regulator Etv2 act in a hierarchical fashion to drive endocardial development. Second, it would shed light on the timing of endocardial specification, providing clear evidence that the endothelial program is not initiated until after Nkx2-5 begins to function in the cardiac crescent.

\section{Implications}

The endocardium plays several well-established roles in the development of the heart. It serves as the source of mesenchymal cells in the endocardial cushions that give rise to the structural elements of the cardiac valves as well as the atrial and membranous ventricular septa. It also participates in less well-appreciated ways in other aspects of cardiovascular development, including the formation of the cardiac conduction system and in proper myocardial trabeculation $[41,60,65]$. Given the toll of pediatric disease represented by valvular abnormalities, septal defects, conduction system abnormalities, and ventricular noncompaction, the role of the endocardium during development is critically important clinically. Thus, a more detailed appreciation of the molecular mechanisms of endocardial development is essential to gain a more nuanced understanding of human developmental defects and eventually may offer the hope of developing novel therapeutic strategies for congenital heart defects.

Open Access This article is distributed under the terms of the Creative Commons Attribution Noncommercial License which permits any noncommercial use, distribution, and reproduction in any medium, provided the original author(s) and source are credited.

\section{References}

1. Abu-Issa R, Kirby ML (2007) Heart field: from mesoderm to heart tube. Annu Rev Cell Dev Biol 23:45-68

2. Akazawa H, Komuro I (2005) Cardiac transcription factor Csx/ Nkx2-5: its role in cardiac development and diseases. Pharmacol Ther 107:252-268

3. al Moustafa AE, Chalifour LE (1993) Immortal cell lines isolated from heart differentiate to an endothelial cell lineage in the presence of retinoic acid. Cell Growth Differ 4:841-847

4. Baldwin HS (1996) Early embryonic vascular development. Cardiovasc Res 31(Spec Iss):E34-E45

5. Brand T (2003) Heart development: molecular insights into cardiac specification and early morphogenesis. Dev Biol 258:1-19

6. Brutsaert DL, De Keulenaer GW, Fransen P, Mohan P, Kaluza GL, Andries LJ, Rouleau JL, Sys SU (1996) The cardiac endothelium: functional morphology, development, and physiology. Prog Cardiovasc Dis 39:239-262

7. Bu L, Jiang X, Martin-Puig S, Caron L, Zhu S, Shao Y, Roberts DJ, Huang PL, Domian IJ, Chien KR (2009) Human ISL1 heart progenitors generate diverse multipotent cardiovascular cell lineages. Nature 460:113-117

8. Bussmann J, Bakkers J, Schulte-Merker S (2007) Early endocardial morphogenesis requires. Sc1/Tal1 PLoS Genet 3:e140

9. Cai CL, Liang X, Shi Y, Chu PH, Pfaff SL, Chen J, Evans S (2003) Isl1 identifies a cardiac progenitor population that proliferates prior to differentiation and contributes a majority of cells to the heart. Dev Cell 5:877-889

10. Charron F, Nemer M (1999) GATA transcription factors and cardiac development. Semin Cell Dev Biol 10:85-91

11. Cohen-Gould L, Mikawa T (1996) The fate diversity of mesodermal cells within the heart field during chicken early embryogenesis. Dev Biol 177:265-273

12. Combs MD, Yutzey KE (2009) Heart valve development: regulatory networks in development and disease. Circ Res 105:408421

13. de la Pompa JL, Timmerman LA, Takimoto H, Yoshida H, Elia AJ, Samper E, Potter J, Wakeham A, Marengere L, Langille BL, Crabtree GR, Mak TW (1998) Role of the NF-ATc transcription 
factor in morphogenesis of cardiac valves and septum. Nature 392:182-186

14. De Val S, Black BL (2009) Transcriptional control of endothelial cell development. Dev Cell 16:180-195

15. De Val S, Chi NC, Meadows SM, Minovitsky S, Anderson JP, Harris IS, Ehlers ML, Agarwal P, Visel A, Xu SM, Pennacchio LA, Dubchak I, Krieg PA, Stainier DY, Black BL (2008) Combinatorial regulation of endothelial gene expression by Ets and Forkhead transcription factors. Cell 135:1053-1064

16. Eisenberg CA, Bader D (1995) QCE-6: a clonal cell line with cardiac myogenic and endothelial cell potentials. Dev Biol 167:469-481

17. Ema M, Takahashi S, Rossant J (2006) Deletion of the selection cassette, but not cis-acting elements, in targeted Flk1-lacZ allele reveals Flk1 expression in multipotent mesodermal progenitors. Blood 107:111-117

18. Fehling HJ, Lacaud G, Kubo A, Kennedy M, Robertson S, Keller G, Kouskoff V (2003) Tracking mesoderm induction and its specification to the hemangioblast during embryonic stem cell differentiation. Development 130:4217-4227

19. Ferdous A, Caprioli A, Iacovino M, Martin CM, Morris J, Richardson JA, Latif S, Hammer RE, Harvey RP, Olson EN, Kyba M, Garry DJ (2009) Nkx2-5 transactivates the Ets-related protein 71 gene and specifies an endothelial/endocardial fate in the developing embryo. Proc Natl Acad Sci USA 106:814-819

20. Flamme I, Frolich T, Risau W (1997) Molecular mechanisms of vasculogenesis and embryonic angiogenesis. J Cell Physiol 173:206-210

21. Gering M, Yamada Y, Rabbitts TH, Patient RK (2003) Lmo2 and Scl/Tal1 convert non-axial mesoderm into haemangioblasts which differentiate into endothelial cells in the absence of Gata1. Development 130:6187-6199

22. Harvey RP (1996) NK-2 homeobox genes and heart development. Dev Biol 178:203-216

23. Harvey RP, Lai D, Elliott D, Biben C, Solloway M, Prall O, Stennard F, Schindeler A, Groves N, Lavulo L, Hyun C, Yeoh T, Costa M, Furtado M, Kirk E (2002) Homeodomain factor Nkx25 in heart development and disease. Cold Spring Harb Symp Quant Biol 67:107-114

24. Hirschi KK, D'Amore PA (1996) Pericytes in the microvasculature. Cardiovasc Res 32:687-698

25. Hirschi KK, D'Amore PA (1997) Control of angiogenesis by the pericyte: molecular mechanisms and significance. Exs 79:419428

26. Huber TL, Kouskoff V, Fehling HJ, Palis J, Keller G (2004) Haemangioblast commitment is initiated in the primitive streak of the mouse embryo. Nature 432:625-630

27. Hutson MR, Kirby ML (2007) Model systems for the study of heart development and disease. Cardiac neural crest and conotruncal malformations. Semin Cell Dev Biol 18:101-110

28. Ishii Y, Langberg J, Rosborough K, Mikawa T (2009) Endothelial cell lineages of the heart. Cell Tissue Res 335:67-73

29. Jaffredo T, Gautier R, Eichmann A, Dieterlen-Lièvre F (1998) Intraaortic hemopoietic cells are derived from endothelial cells during ontogeny. Development 125:4575-4583

30. Kattman SJ, Huber TL, Keller GM (2006) Multipotent flk-1+ cardiovascular progenitor cells give rise to the cardiomyocyte, endothelial, and vascular smooth muscle lineages. Dev Cell $11: 723-732$

31. Komuro I, Izumo S (1993) Csx: a murine homeobox-containing gene specifically expressed in the developing heart. Proc Natl Acad Sci USA 90:8145-8149

32. Kouskoff V, Lacaud G, Schwantz S, Fehling HJ, Keller G (2005) Sequential development of hematopoietic and cardiac mesoderm during embryonic stem cell differentiation. Proc Natl Acad Sci USA 102:13170-13175
33. Lee RK, Stainier DY, Weinstein BM, Fishman MC (1994) Cardiovascular development in the zebrafish. II. Endocardial progenitors are sequestered within the heart field. Development 120:3361-3366

34. Lee D, Park C, Lee H, Lugus JJ, Kim SH, Arentson E, Chung YS, Gomez G, Kyba M, Lin S, Janknecht R, Lim DS, Choi K (2008) ER71 acts downstream of BMP, Notch, and Wnt signaling in blood and vessel progenitor specification. Cell Stem Cell 2:497507

35. Linask KK, Lash JW (1993) Early heart development: dynamics of endocardial cell sorting suggests a common origin with cardiomyocytes. Dev Dyn 196:62-69

36. Lints TJ, Parsons LM, Hartley L, Lyons I, Harvey RP (1993) Nkx-25: a novel murine homeobox gene expressed in early heart progenitor cells and their myogenic descendants. Development 119:969

37. Lyons I, Parsons LM, Hartley L, Li R, Andrews JE, Robb L, Harvey RP (1995) Myogenic and morphogenetic defects in the heart tubes of murine embryos lacking the homeo box gene Nkx2-5. Genes Dev 9:1654-1666

38. Maschhoff KL, Baldwin HS (2000) Molecular determinants of neural crest migration. Am J Med Genet 97:280-288

39. Masino AM, Gallardo TD, Wilcox CA, Olson EN, Williams RS, Garry DJ (2004) Transcriptional regulation of cardiac progenitor cell populations. Circ Res 95:389-397

40. Meilhac SM, Kelly RG, Rocancourt D, Eloy-Trinquet S, Nicolas JF, Buckingham ME (2003) A retrospective clonal analysis of the myocardium reveals two phases of clonal growth in the developing mouse heart. Development 130:3877-3889

41. Mikawa T, Hurtado R (2007) Development of the cardiac conduction system. Semin Cell Dev Biol 18:90-100

42. Mikawa T, Gourdie RG, Takebayashi-Suzuki K, Kanzawa N, Hyer J, Pennisi DJ, Poma CP, Shulimovich M, Diaz KG, Layliev J, Prasad A (2003) Induction and patterning of the Purkinje fibre network. Novartis Found Symp 250:142-153 discussion 153146, 276-149

43. Misfeldt AM, Boyle SC, Tompkins KL, Bautch VL, Labosky PA, Baldwin HS (2009) Endocardial cells are a distinct endothelial lineage derived from Flk1+ multipotent cardiovascular progenitors. Dev Biol 333:78-89

44. Moretti A, Caron L, Nakano A, Lam JT, Bernshausen A, Chen Y, Qyang Y, Bu L, Sasaki M, Martin-Puig S, Sun Y, Evans SM, Laugwitz KL, Chien KR (2006) Multipotent embryonic isl1+ progenitor cells lead to cardiac, smooth muscle, and endothelial cell diversification. Cell 127:1151-1165

45. Moses KA, DeMayo F, Braun RM, Reecy JL, Schwartz RJ (2001) Embryonic expression of an Nkx2-5/Cre gene using ROSA26 reporter mice. Genesis 31:176-180

46. Motoike T, Markham DW, Rossant J, Sato TN (2003) Evidence for novel fate of Flk1+ progenitor: contribution to muscle lineage. Genesis 35:153-159

47. Nemer G, Nemer M (2002) Cooperative interaction between GATA5 and NF-ATc regulates endothelial-endocardial differentiation of cardiogenic cells. Development 129:4045-4055

48. Park C, Ma YD, Choi K (2005) Evidence for the hemangioblast. Exp Hematol 33:965-970

49. Patan S (2000) Vasculogenesis and angiogenesis as mechanisms of vascular network formation, growth and remodeling. J Neurooncol 50:1-15

50. Patan S (2004) Vasculogenesis and angiogenesis. Cancer Treat Res 117:3-32

51. Person AD, Klewer SE, Runyan RB (2005) Cell biology of cardiac cushion development. Int Rev Cytol 243:287-335

52. Puri MC, Partanen J, Rossant J, Bernstein A (1999) Interaction of the TEK and TIE receptor tyrosine kinases during cardiovascular development. Development 126:4569-4580 
53. Ranger AM, Grusby MJ, Hodge MR, Gravallese EM, de la Brousse FC, Hoey T, Mickanin C, Baldwin HS, Glimcher LH (1998) The transcription factor NF-ATc is essential for cardiac valve formation. Nature 392:186-190

54. Saga Y, Kitajima S, Miyagawa-Tomita S (2000) Mesp1 expression is the earliest sign of cardiovascular development. Trends Cardiovasc Med 10:345-352

55. Schultheiss TM, Burch JB, Lassar AB (1997) A role for bone morphogenetic proteins in the induction of cardiac myogenesis. Genes Dev 11:451-462

56. Schwartz RJ, Olson EN (1999) Building the heart piece by piece: modularity of cis-elements regulating $\mathrm{Nkx} 2-5$ transcription. Development 126:4187-4192

57. Snarr BS, Kern CB, Wessels A (2008) Origin and fate of cardiac mesenchyme. Dev Dyn 237:2804-2819

58. Stainier DY, Lee RK, Fishman MC (1993) Cardiovascular development in the zebrafish. I. Myocardial fate map and heart tube formation. Development 119:31-40

59. Stainier DY, Weinstein BM, Detrich HW III, Zon LI, Fishman MC (1995) Cloche, an early acting zebrafish gene, is required by both the endothelial and hematopoietic lineages. Development 121:3141-3150

60. Stankunas K, Hang CT, Tsun ZY, Chen H, Lee NV, Wu JI, Shang C, Bayle JH, Shou W, Iruela-Arispe ML, Chang CP (2008) Endocardial Brg1 represses ADAMTS1 to maintain the microenvironment for myocardial morphogenesis. Dev Cell 14:298-311

61. Sumanas S, Gomez G, Zhao Y, Park C, Choi K, Lin S (2008) Interplay among Etsrp/ER71, Scl, and Alk8 signaling controls endothelial and myeloid cell formation. Blood 111:4500-4510
62. Tanaka M, Chen Z, Bartunkova S, Yamasaki N, Izumo S (1999) The cardiac homeobox gene Csx/Nkx25 lies genetically upstream of multiple genes essential for heart development. Development 126:1269-1280

63. Thomas M, Augustin HG (2009) The role of the Angiopoietins in vascular morphogenesis. Angiogenesis 12:125-137

64. Verzi MP, McCulley DJ, De Val S, Dodou E, Black BL (2005) The right ventricle, outflow tract, and ventricular septum comprise a restricted expression domain within the secondary/anterior heart field. Dev Biol 287:134-145

65. Wagner M, Siddiqui MAQ (2007) Signal transduction in early heart development (II): ventricular chamber specification, trabeculation, and heart valve formation. Exp Biol Med (Maywood) 232:866-880

66. Wei Y, Mikawa T (2000) Fate diversity of primitive streak cells during heart field formation in ovo. Dev Dyn 219:505-513

67. Xiong JW (2008) Molecular and developmental biology of the hemangioblast. Dev Dyn 237:1218-1231

68. Zeisberg EM, Ma Q, Juraszek AL, Moses K, Schwartz RJ, Izumo $\mathrm{S}, \mathrm{Pu}$ WT (2005) Morphogenesis of the right ventricle requires myocardial expression of Gata4. J Clin Invest 115:1522-1531

69. Zovein AC, Hofmann JJ, Lynch M, French WJ, Turlo KA, Yang Y, Becker MS, Zanetta L, Dejana E, Gasson JC, Tallquist MD, Iruela-Arispe ML (2008) Fate tracing reveals the endothelial origin of hematopoietic stem cells. Cell Stem Cell 3:625-636 